\title{
First Measurement of the Linear Polarization of Radiative Electron Capture Transitions
}

\author{
S. Tashenov, ${ }^{1,2}$ Th. Stöhlker, ${ }^{1,2}$ D. Banaś ${ }^{3}$ K. Beckert, ${ }^{1}$ P. Beller, ${ }^{1}$ H. F. Beyer, ${ }^{1}$ F. Bosch, ${ }^{1}$ S. Fritzsche, ${ }^{4}$ A. Gumberidze, ${ }^{1,2}$ \\ S. Hagmann, ${ }^{1,2}$ C. Kozhuharov, ${ }^{1}$ T. Krings,${ }^{5}$ D. Liesen, ${ }^{1}$ F. Nolden, ${ }^{1}$ D. Protic, ${ }^{5}$ D. Sierpowski, ${ }^{6}$ U. Spillmann, ${ }^{1,2}$ \\ M. Steck, ${ }^{1}$ and A. Surzhykov ${ }^{4}$ \\ ${ }^{1}$ Gesellschaft für Schwerionenforschung 64291 Darmstadt, Germany \\ ${ }^{2}$ Institut für Kernphysik University of Frankfurt 60486 Frankfurt, Germany \\ ${ }^{3}$ Institute of Physics Swietokrzyska Academy 25-406 Kielce, Poland \\ ${ }^{4}$ University of Kassel Heinrich-Plett-Strasse 4034132 Kassel, Germany \\ ${ }^{5}$ Institute for Nuclear Physics Forschungszentrum FZ-Jülich, Germany \\ ${ }^{6}$ Institute of Physics Jagiellonian University 30-059 Cracow, Poland
}

(Received 23 November 2005; published 28 November 2006)

\begin{abstract}
For radiative electron capture into the $K$ shell of bare uranium ions, a study of the polarization properties has been performed. For this purpose a position sensitive germanium detector has been used as an efficient Compton polarimeter. This enabled us to measure the degree of linear polarization by analyzing Compton scattering inside the detector and to determine the orientation of the polarization plane. Depending on the observation angle and the beam energy used, the radiation is found to be linearly polarized by up to $80 \%$. In all cases studied, the plane of polarization coincides with the collision plane. The results will be discussed in the context of rigorous relativistic calculations, showing that relativistic effects tend to lead to a depolarization of the radiation emitted.
\end{abstract}

Radiative capture of free or quasifree electrons (REC) into high- $Z$ ions has been found to provide a wealth of information on the electron-photon interaction in the presence of strong fields [1,2]. Most important, today, REC is an established tool for studying the time-reversed process, the elementary photoeffect, for one- and few-electron ions at high $Z$ which can otherwise not be addressed in experiments [2]. As a consequence, most properties of the REC were the subject of detailed experimental and theoretical studies (see [2-5] and references therein). However, a very important aspect of linear polarization so far has not been the subject of experimental investigations although the results of the angular-differential studies already indicate that REC may exhibit distinctive polarization features. Even for the time-reversed process and neutral heavy elements only very few experimental data are available $[6,7]$. There, the results are strongly affected by electron scattering in the target and except at very high photon energies (above $1 \mathrm{MeV}$ ) an unambiguous interpretation of the data is hampered by distortion effects caused by the solid targets used [7]. In contrast, these effects are completely absent in REC studies. Moreover, the polarization features of $K$-REC radiation have recently attracted particular attention because of its predicted sensitivity to a possible spin polarization of the particles involved in the collision (electrons or ions) [8]. As a consequence, the detection of the linear polarization of $K$-REC might be applied as an important tool for the control and diagnostics of the degree of the spin polarization of heavy ion beams confined in storage rings. Currently, such a technique is much needed for experiments aiming on a detection of parity nonconservation effects in highly charged ions and for the search of the electric dipole moment of heavy nuclei as proposed for a test of the standard model [9-11]. In this context, we like to emphasize that REC into the $K$ shell is the most important charge exchange channel for heavy ions in collisions with light target atoms and, therefore, represents an intense source of radiation.

In this Letter we report the first experimental study of the (linear) photon polarization of the REC radiation in ion-atom collisions. The investigations were conducted for bare uranium ions at various energies in the range between 100 and $400 \mathrm{MeV} / u$, using nitrogen as a target. A position sensitive germanium pixel detector [12] as Compton polarimeter, exploiting the polarization sensitivity of the Compton scattering process, was utilized. The principles of this technique are well established in $\gamma$-ray spectroscopy [13], the application of a pixel array for polarimetry of energetic photons has the advantage that it provides a high detection efficiency in combination with a good energy resolution and position sensitivity $[14,15]$. This novel experimental approach for the investigation of the projectile $\mathrm{x}$-ray emission characteristics in heavy ion-atom collisions enabled us to measure for the very first time the polarization plane and the degree of (linear) polarization for $K$-REC radiation.

Following the Klein-Nishina formula, the differential cross section for Compton scattering of a photon with initial energy $\hbar \omega$ is given by

$$
\frac{d \sigma}{d \Omega} \propto\left(\frac{\hbar \omega^{\prime}}{\hbar \omega}\right)^{2}\left(\frac{\hbar \omega^{\prime}}{\hbar \omega}+\frac{\hbar \omega}{\hbar \omega^{\prime}}-2 \sin ^{2} \theta_{C} \cos ^{2} \varphi\right),
$$

where $\hbar \omega^{\prime}$ denotes the energy of the scattered photon and $\theta_{C}$ and $\varphi$ are the angles of the scattered photon with respect 
to the propagation direction of the incident radiation and its polarization plane (indicated by the vector in Fig. 1), respectively. As a consequence, for linearly polarized radiation, Compton scattering results in an almost dipolar angular distribution of the scattered photons with a maximum perpendicular to the polarization direction [13]. Experimentally, this distribution can be accessed by position sensitive detectors exploiting the fact that for our energies of interest the recoil electron is always stopped in the close vicinity to the initial interaction point. Therefore, an unambiguous identification of Compton events is attained by a coincident registration of the position and the energy for both the Compton recoil electron and the Compton scattered photon. Note, that the energy deposition, $\Delta E$, of the Compton recoil electron and the energy of the Compton scattered photon must add up always to the energy of the incident radiation.

For the experiment REC transitions produced in collisions of $\mathrm{U}^{92+} \rightarrow \mathrm{N}_{2}$ were studied at the internal target of the ESR storage ring at the energies of 400, 200, 132, and $98 \mathrm{MeV} / u$, respectively. In case of energies lower than $400 \mathrm{MeV} / u$, the deceleration mode of the ESR was applied. For the details of ion beam preparation and the experimental environment at the ESR we refer to the literature [2]. At the high beam energy of $400 \mathrm{MeV} / u$, the polarimeter came into operation at the gas-jet scattering chamber of the ESR at observation angles of $\theta_{\mathrm{lab}}=90^{\circ}$ and $\theta_{\text {lab }}=60^{\circ}$, respectively. For the lower beam energies, this device was installed at $60^{\circ}$ observation angle only. Each of the 16 individual pixels (pixel size: $7 \times 7 \mathrm{~mm}^{2}$ ), arranged in a $4 \times 4$ matrix, was equipped with an individual electronic readout using standard analogue and digital electronics. This electronic setup enabled us to record energy and time signals separately for each of the pixels with a typical energy resolution of close to 2 at $122 \mathrm{keV}$. In addition to the coincident registration of events occurring simultaneously in two of the pixels, $\mathrm{x}$ rays were also recorded in coincidence with the down-charged uranium



FIG. 1 (color online). Geometry of the detector arrangement at the ESR jet target. The collision plane is defined by the ion beam direction and the propagation direction of the emitted REC photon. For details compare the text. ions as produced by REC from the target electrons. Downcharged $\mathrm{U}^{91+}$ particles were registered by a particle detector installed behind the first dipole magnet downstream of the gas-jet target.

In Fig. 1 the geometry of the detector arrangement is displayed with respect to the collision plane as defined by the projectile ion beam and the direction of the emitted REC photons. As an example, a scatter plot of events registered in coincidence between two pixels is displayed in the top part of Fig. 2. These data were taken under $90^{\circ}$ for $400 \mathrm{MeV} / u \mathrm{U}^{92+} \rightarrow \mathrm{N}_{2}$ collisions. In the figure, the large number of events in the diagonal corresponds to events with a (constant) sum energy equal to the $K$-REC transition, i.e., for $E_{K \text {-REC }}=\Delta E+\hbar \omega^{\prime}$. It is worthwhile to mention that, for our initial energies $\left(E_{K \text {-REC }} \approx 250 \mathrm{keV}\right)$, the condition $\Delta E<\hbar \omega^{\prime}$ is always fulfilled. The latter also explains the two well-separated maxima visible within the scatter plot. One pixel measures the energy of the Compton recoil electron $(\Delta E)$ and the other one records the energy of the scattered photon $\left(\hbar \omega^{\prime}\right)$ or vice versa. We note, that the less pronounced and washed out diagonal visible at the sum energy of about $150 \mathrm{keV}$ is caused by REC into the $L$ and $M$ shell of the projectile. Beside the scatter plot representation, it is instructive to display the corresponding


FIG. 2 (color online). Top: Scatter plot for coincident Compton events as observed for $400 \mathrm{MeV} / u \mathrm{U}^{92+} \rightarrow \mathrm{N}_{2}$ collisions at an observation angle of $90^{\circ}$, the $K$-REC photon energy amounts to $240 \mathrm{keV}$. Bottom: $\mathrm{x}$-ray spectra reconstructed from the sum of the electron recoil energy $(\Delta E)$ and the energy $\hbar \omega^{\prime}$ of the Compton scattered photon. While the shaded area depicts the sum energy spectrum for scattering within the collision plane $\left(\varphi=0^{\circ}\right)$, the white area $\left(\varphi=90^{\circ}\right)$ refers to scattering orthogonal to it. 
sum energy spectra. In the bottom part of Fig. 2, sample sum energy spectra $\left(\Delta E+\hbar \omega^{\prime}\right)$ for the observation angle of $90^{\circ}$ at $400 \mathrm{MeV} / u \mathrm{U}^{92+} \rightarrow \mathrm{N}_{2}$ collisions are shown. While the shaded area depicts the sum energy spectrum for scattering within the collision plane $\left(\varphi=0^{\circ}\right)$, the white area $\left(\varphi=90^{\circ}\right)$ refers to scattering orthogonal to it. Following Fig. 2, Compton scattering of the $K$-REC radiation perpendicular to the collision plane appears strongly enhanced compared to the scattering into the parallel direction and the effect of polarization shows up particularly pronounced for the intensity ratio. We note, that within the detector the scattering angle $\varphi$ is defined by the relative position of the two pixels involved. Because of the matrix structure of the pixel arrangement there is always a further combination of two pixels with the angle $\varphi+90^{\circ}$ which exhibits the same scattering geometry (solid angle, distance). Therefore, experimentally, this fact allows for an internal intensity normalization $I_{\varphi} / I_{\varphi+90^{\circ}}$ which reduces substantially the influence of possible systematic uncertainties. Here the most important effects to mention are the absorption efficiency and the solid angle correction for the scattered photons, both effects which cancel out completely for the case of the intensity ratio. For the particular case of the $90^{\circ}$ observation angle at $400 \mathrm{MeV} / u$, we plot in Fig. 3 the normalized intensity distribution $I_{\varphi} / I_{\varphi+90^{\circ}}$ as function of the scattering angle $\varphi$ (compare solid points) [16]. In the figure, a strong intensity variation for Compton scattering as function of the scattering angle is observed. Note, because for neighboring pixels coincident events might have also been produced by charge splitting or electronic cross talk, such pixel combinations were not considered.

The degree of linear polarization was derived on the basis of the following formula:

$$
I_{\varphi} / I_{\varphi+90^{\circ}}=\frac{d \sigma}{d \Omega_{\varphi}} / \frac{d \sigma}{d \Omega_{\varphi+90^{\circ}}} .
$$

Here, the differential cross section $d \sigma / d \Omega_{\varphi}$ corresponds

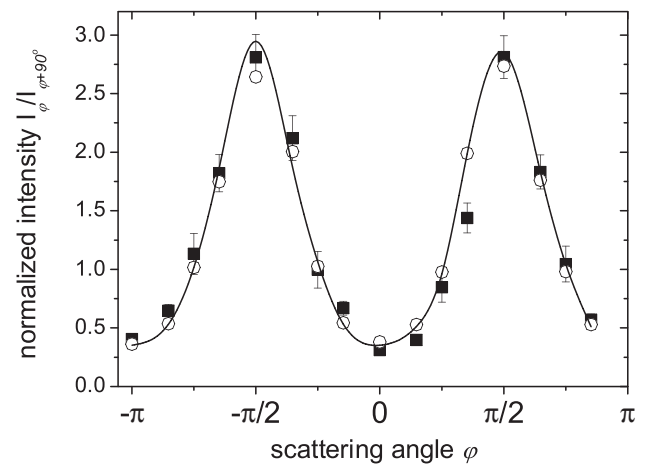

FIG. 3. Normalized intensity distribution for Compton scattered $K$-REC radiation $I_{\varphi} / I_{\varphi+90^{\circ}}$ as function of the scattering angle $\varphi\left(90^{\circ}\right.$ observation angle at $\left.400 \mathrm{MeV} / u\right)$ (for details compare text). to the Klein-Nishina formula [compare Eq. (1)], except for the $\cos ^{2} \varphi$ term which was replaced by the expression $P(d)=\frac{1}{2}(1-d)+d \cos ^{2} \varphi$ in order to account for the partially polarized nature of the photon beam where $d$ denotes the fraction of linearly polarized photons. Note for $d=0$ and $d=1$, the corresponding Klein-Nishina formulas for unpolarized and completely polarized photon beams are restored. In addition, the theoretical distribution was convoluted by the detector response function in order to account for detector specific effects. The most important correction introduced here was due to the finite size of the detector segmentation. For completeness, also the Compton profile of the electrons bound in the germanium atoms, Rayleigh scattering, and the uncertainty in the depletion depth of the detector were considered (14 \pm $1 \mathrm{~mm}$ ). Information about the detector response was derived by laboratory measurements using standard radioactive sources $\left({ }^{133} \mathrm{Ba},{ }^{169} \mathrm{Yb}\right.$ and $\left.{ }^{182} \mathrm{Ta}\right)$ and by numerical simulations. The overall uncertainty associated with these corrections can be estimated to be of the order of $3 \%$ or less. Finally, by taking into account the detector response, Eq. (2) was adjusted to the experimental data by the least square method using the MINUIT software package [17]. Within this algorithm, $d$ was treated as a free fit parameter. As a redundancy check, the response of the detector to polarized radiation was computed using the wellestablished EGS4 Monte Carlo detector simulation code $[18,19]$ and compared with the experimental data. In Fig. 3, as an example, we compare the experimental intensity distribution with the result obtained by the procedure discussed above (solid line), which corresponds to a linear polarization of $79 \% \pm 8 \%$ for the observed $K$-REC radiation. The results of the EGS4 simulation code for the detector response on $79 \%$ linearly polarized radiation are also given (see open circles in Fig. 3). As seen in the figure, the experimental data turn out to be in good agreement with the simulation, showing pronounced maxima perpendicular to the collision plane whereas minima occur parallel to this plane. The latter finding is consistent with the fact that both particles involved into the collision, the electron and the ion, are not spin polarized [8].

Table I shows the degree of linear polarization for the various ion beam energies and observation angles $\left(\theta_{\mathrm{lab}}\right)$ used in the experiment along with the corresponding emis-

TABLE I. Experimental results for the degree of the $K$-REC polarization compared to theory.

\begin{tabular}{cccccc}
\hline \hline $\begin{array}{c}\text { Energy } \\
(\mathrm{MeV} / \mathrm{u})\end{array}$ & $\begin{array}{c}\theta_{\mathrm{lab}} \\
(\mathrm{deg})\end{array}$ & $\begin{array}{c}\theta_{\mathrm{em}} \\
(\mathrm{deg})\end{array}$ & $\begin{array}{c}\hbar \omega \\
(\mathrm{keV})\end{array}$ & $\begin{array}{c}\text { Experiment } \\
(\%)\end{array}$ & $\begin{array}{c}\text { Theory [3-5] } \\
(\%)\end{array}$ \\
\hline 400 & 90 & 135.6 & 246 & $79 \pm 8$ & 83.5 \\
400 & 60 & 109.5 & 382 & $61 \pm 12$ & 69.2 \\
190 & 60 & 94.5 & 270 & $72 \pm 5$ & 80.3 \\
132 & 60 & 88.7 & 236 & $83 \pm 5$ & 83.8 \\
98 & 60 & 84.6 & 214 & $85 \pm 7$ & 85.9 \\
\hline \hline
\end{tabular}




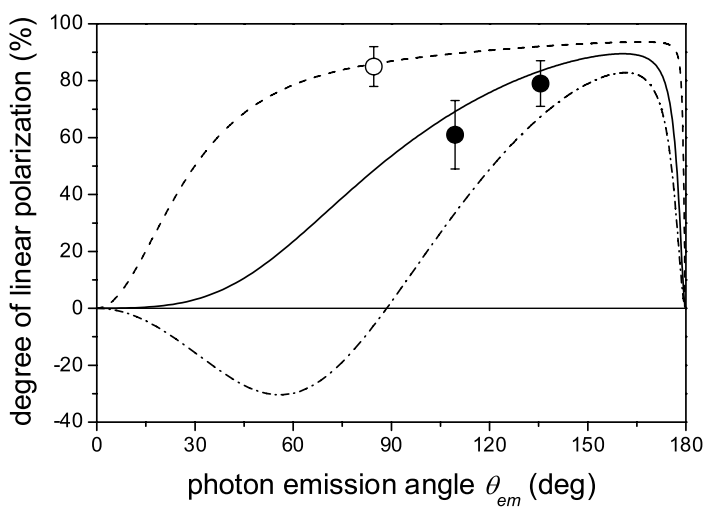

FIG. 4 (color online). Linear photon polarization as observed for $\mathrm{U}^{92+} \rightarrow \mathrm{N}_{2}$ collisions as function of the photon emission angle (moving system): $400 \mathrm{MeV} / u$ solid circles; $98 \mathrm{MeV} / u$ open circle. For comparison, the results of rigorous relativistic calculations are given: $98 \mathrm{MeV} / u$ dashed line; $400 \mathrm{MeV} / u$ full line; $800 \mathrm{MeV} / u$ dashed-dotted line [3-5].

sion angles in the moving frame $\left(\theta_{\mathrm{em}}\right)$ in comparison with theoretical predictions [3-5]. The experimental uncertainties are mainly of statistical nature (5\% and larger). As seen from Table I, the $K$-REC radiation appears to be strongly polarized within the collision plane. However, the degree of linear polarization becomes smaller as the projectile energy is increased, an effect which is seen, in particular, at the observation angle $\theta_{\mathrm{lab}}=60^{\circ}$. In fact, a very similar decrease of the polarization has been predicted recently in rigorous relativistic calculations [3-5]. In these computations, attention was paid to the nondipole contributions to the electron-photon interaction, which become more pronounced for the higher collision energies and, hence, lead to a depolarization of the emitted radiation. As seen from Fig. 4, the nondipolar effects are expected to reduce the degree of linear polarization of the $K$-REC photons over all photon emission angles in the moving frame $\theta_{\mathrm{em}}$ when compared with the nonrelativistic theory where the degree of polarization is always $100 \%$. Here it is instructive to point out the consistency of our findings with the predictions made for the inverse reaction, the photoionization of the $1 s$ state in uranium, as it has been reported (compare Fig. 3 in Ref. [20]). Also these computations show a decrease in polarization with increasing energy as higher multipole and relativistic effects come into play. Finally, we note that for high collisions energies $T_{p} \geq 500 \mathrm{MeV} / u$ and for a photon emission in forward direction a fully relativistic treatment of the electron-photon interaction predicts a negative (degree of) linear polarization already known from theoretical photoionization studies [21,22].

In summary, we reported a first experimental study of the linear polarization of REC transitions. For the case of the $K$ shell of bare uranium, the radiation is found to be strongly polarized, depending on both the emission angle and the collision energy. The agreement found with rigorous relativistic calculations also indicates that one may even expect a $100 \%$ polarization of the radiation for the case of low- and medium- $Z$ ions at moderate nonrelativistic energies. To the best of our knowledge, there is no other atomic collision process known which exhibits such an extraordinary degree of polarization for a monochromatic hard $\mathrm{x}$-ray transition.

[1] J. Eichler and W.E. Meyerhof Relativistic Atomic Collisions (Academic, San Diego, 1995).

[2] Th. Stöhlker et al., Phys. Rev. Lett. 86, 983 (2001).

[3] A. Surzhykov, S. Fritzsche, and Th. Stöhlker, Phys. Lett. A 289, 213 (2001).

[4] A. Surzhykov et al., Phys. Rev. A 68, 022710 (2003).

[5] J. Eichler and A. Ichihara, Phys. Rev. A 65, 052716 (2002).

[6] A. Logan, J. Phys. A 4, 346 (1971).

[7] A. Logan, R. T. Jones, A. Ljubicic, W. R. Dixon, and R. S. Storey, Phys. Rev. A 10, 532 (1974).

[8] A. Surzhykov, S. Fritzsche, Th. Stöhlker, and S. Tashenov, Phys. Rev. Lett. 94, 203202 (2005).

[9] L. N. Labzowsky, A. V. Nefiodov, G. Plunien, G. Soff, R. Marrus, and D. Liesen, Phys. Rev. A 63, 054105 (2001).

[10] A. V. Nefiodov, L. N. Labzowsky, D. Liesen, G. Plunien, and G. Soff, Phys. Lett. B 534, 52 (2002).

[11] Conceptual Design Report: An International Accelerator Facility for Beams of Ions and Antiprotons, edited by W. Henning, GSI (2001).

[12] This detector has been provided by IKP Forschungszentrum Jülich, 2000, see also A. Hamacher et al., JülSpez-403, ISSN 0170-8937, 117 (1987).

[13] Kai Siegbahn Alpha-, Beta- and Gamma-Ray Spectroscopy (North-Holland, Amsterdam, 1968), Vol. 2.

[14] R. A. Kroeger, W. N. Johnson, J. D. Kurfess, and B. F. Philips, Nucl. Instrum. Methods Phys. Res., Sect. A 436, 165 (1999).

[15] J.H. Lee and C. S. Lee, Nucl. Instrum. Methods Phys. Res., Sect. A 506, 125 (2003).

[16] S. Tashenov, Ph.D. thesis, University of Frankfurt, 2005.

[17] F. James and M. Roos, Comput. Phys. Commun. 10, 343 (1975).

[18] W. R. Nelson, H. Hirayama, and D. W. O. Rogers, EGS4 Code System, Stanford Linear Accelerator Center, Stanford, CA, Report No. SLAC-265, 1985.

[19] H. Hirayama and Y. Namito, KEK Internal Report No. 2000-4, 2000.

[20] Y. S. Kim, I. B. Goldberg, and R. H. Pratt, Phys. Rev. A 45, 4542 (1992).

[21] U. Fano, K. W. McVoy, and J. R. Albers, Phys. Rev. 116, 1159 (1959).

[22] R. H. Pratt, R. D. Levee, R. L. Pexton, and W. Aron, Phys. Rev. 134, A916 (1964). 